\title{
Aprotinin Reduces Cardiac Troponin I Release and Inhibits Apoptosis of Polymorphonuclear Cells During Off-Pump Coronary Artery Bypass Surgery
}

\author{
Christophe Bert, MD, PhD, * Frederik De Buck, MD,* Paul Sergeant, MD, PhD, † Jan Van Hemelrijck, MD, PhD,* \\ Ahmad Kasran, MD, PhD, $\neq$ Victor Van Duppen, $\S$ Jan Ceuppens, MD, PhD, $\neq$ Bart Meyns, MD, PhD, $\dagger$ \\ Michel Delforge, MD, PhD, $\S$ and Patrick Wouters, MD, PhD*
}

\begin{abstract}
Objectives: In addition to blood-sparing effects, aprotinin may have cardioprotective and anti-inflammatory effects during cardiopulmonary bypass-assisted cardiac surgery. In this study, the authors examined whether aprotinin had cardioprotective and/or anti-inflammatory effects in patients undergoing off-pump coronary artery bypass grafting.

Design: A prospective randomized clinical trial.

Setting: University hospital.

Participants: Fifty patients were randomized to control ( $n=25)$ or aprotinin treatment $(n=25)$ groups.

Interventions: Aprotinin was given as a loading dose $(2 \times$ $10^{6} \mathrm{KIU}$ ) followed by a continuous infusion at $5 \times 10^{5} \mathrm{KIU} / \mathrm{h}$ until skin closure.

Measurements and Main Results: Blood samples for cardiac troponin I; interleukin-6, interleukin-8, and interleukin10; tumor necrosis factor $\alpha$; and elastase were taken after anesthesia induction, completion of revascularization, and 6 hours, 12 hours, and 24 hours after revascularization. Blood
\end{abstract}

B OTH MECHANICAL INJURY during cardiac surgery and ischemia-reperfusion cause inflammation. In vitro experiments report anti-inflammatory effects of aprotinin by multiple mechanisms, including inhibiting nuclear factor kappa-B $(\mathrm{NF} \kappa \mathrm{B})$ activation, protease activated receptor-1 (PAR-1) activation, phospholipase A2 activity, expression of L-selectin, intercellular adhesion molecule-1, and vascular cellular adhesion molecule. ${ }^{1-6}$ Neutrophils mediate inflammation-associated tissue damage by binding to activated endothelial cells and migrating into the surrounding tissues where they release proteases and oxygen free radicals, leading to tissue damage. Inflammatory responses also induce apoptosis of tissue cells, potentially leading to altered organ function, and apoptosis of polymorphonuclear (PMN) cells is a key event in resolving acute inflammatory processes. Jimenez ${ }^{7}$ reported that PMN cells become apoptosis resistant during systemic inflammatory responses prolonging the lifespan of these cells. This phenomenon is thought to contribute to the organ damage observed during systemic inflammatory response syndrome (SIRS) and related syndromes. Although cardiopulmonary bypass (CPB) is a major factor eliciting a generalized inflammatory reaction, inflammation also occurs in off-pump cardiac surgery, be it in a less pronounced way. ${ }^{8-10}$ Aprotinin has been demonstrated to reduce inflammation in CPB-supported cardiac surgery. ${ }^{11-19}$ Whether aprotinin can further reduce inflammation in off-pump

From the Departments of *Anesthesiology, $†$ Cardiac Surgery, and $\S$ Hematology, and $\$$ Laboratory of Immunology, University Hospital Gasthuisberg, Leuven, Belgium.

Address reprint requests to Christophe Bert, MD, PhD, Dienst Anesthesie, OLV Ziekenhuis, Moorselbaan 164, B-9300 Aalst, Belgium. E-mail: christophe.bert@olvz-aalst.be

(C) 2008 Elsevier Inc. All rights reserved.

1053-0770/08/2201-0005\$34.00/0

doi:10.1053/j.jvca.2007.01.018 samples were taken to assess for apoptosis in polymorphonuclear cells. Baseline plasma levels for cardiac troponin I did not differ between groups but were significantly lower in aprotinin-treated patients at the time of revascularization $(p=0.03)$ and 6 hours $(p=0.004)$ and 24 hours $(p=0.03)$ later. Aprotinin significantly reduced apoptosis in polymorphonuclear cells compared with control-treated patients $(p=0.04)$. There were no differences in plasma cytokine or elastase levels between groups.

Conclusions: The authors conclude that aprotinin reduces perioperative cardiac troponin I release and attenuates apoptosis in polymorphonuclear cells but has no significant effects on plasma cytokine levels in patients undergoing off-pump coronary artery bypass graft surgery.

(c) 2008 Elsevier Inc. All rights reserved.

KEY WORDS: off-pump coronary artery bypass graft, aprotinin, inflammation, myocardial protection, neutrophils, apoptosis, blood loss

coronary artery bypass graft (OPCAB) surgery has currently not been investigated. In addition, aprotinin has been reported to have cardioprotective properties during cardiac surgery with CPB by reducing ischemia-reperfusion injury, including reduced cardiac troponin I (cTnI) release in coronary artery bypass graft (CABG) patients. ${ }^{20-24}$ Reducing inflammatory activation during surgical trauma and ischemia-reperfusion could lead to reduced myocardial tissue injury. If, in addition, activated PMN cells would become less resistant on apoptosis, their reduced lifespan could contribute to decreased tissue injury.

Little data are currently available on the potential cardioprotective and/or anti-inflammatory action of aprotinin in OPCAB surgery. The authors performed a prospective randomized clinical trial to evaluate aprotinin's effects in patients undergoing OPCAB surgery using cTnI as a marker of myocardial damage and interleukin (IL)-6, -8, -10, tumor necrosis factor $\alpha$ (TNF- $\alpha)$, PMN elastase, and PMN cell apoptosis as markers of inflammation.

\section{METHODS}

After approval by the institutional ethics committee, 50 patients undergoing elective OPCAB surgery were enrolled in the study after written informed consent was obtained. Fifty consecutive patients were assigned to the control group $(\mathrm{n}=25)$ or to the aprotinin group $(\mathrm{n}=$ $25)$ by a computer-generated randomization list. Exclusion criteria were as follows: redo surgery, ejection fraction $<30 \%$, chronic immunosuppressive therapy (including corticosteroids), recent infection, unstable angina, use of coumarin derivatives and GPIIb-IIIa inhibitors, documented allergy, congenital coagulation disorders, and renal impairment (serum creatinine $>1.5 \mathrm{mg} / \mathrm{dL}$ ).

All patients received standard anesthetic care that included premedication with $0.05 \mathrm{mg} / \mathrm{kg}$ of lorazepam sublingually 1 hour before surgery. Anesthesia induction was with midazolam $(0.03 \mathrm{mg} / \mathrm{kg})$, sufentanil (1-1.5 $\mu \mathrm{g} / \mathrm{kg}$ ), propofol target-controlled infusion (starting 
target concentration of 0.5-1.0 $\mu \mathrm{g} / \mathrm{mL})$, and pancuronium $(0.1 \mathrm{mg} / \mathrm{kg})$. Anesthesia maintenance was with a continuous propofol infusion (up to target concentration of $2.5 \mu \mathrm{g} / \mathrm{mL}$ ) and supplemental sufentanil (8-10 $\mu \mathrm{g} / \mathrm{kg}$ ) and pancuronium. Vaporizers for volatile anesthetics were removed from the ventilator. Patient monitoring included direct arterial pressure and pulmonary artery pressure monitoring. Patients allocated to the aprotinin group received a loading dose of $2 \times 10^{6} \mathrm{KIU}$ before sternotomy, followed by a continuous infusion of $5 \times 10^{5} \mathrm{KIU} / \mathrm{h}$ until wound closure. Heparin was given as anticoagulation to maintain the activated coagulation times $>400$ seconds. All coronary bypass graft anastomoses were made with intracoronary shunts in place. The region to be operated on was immobilized by a regional vacuum stabilizer and, if needed, by an apical suction device. After revascularization was complete heparin was neutralized with protamine (1:1 ratio of active heparin) and final activated coagulation times were generally within $10 \%$ of baseline value. All patients underwent complete revascularization. Suctioned blood was washed (Autolog; Medtronic Inc, Minneapolis, $\mathrm{MN}$ ) and returned to the patient if the collected volume exceeded $800 \mathrm{~mL}$. All patients received routine postoperative care. Blood sugar concentrations were kept between 70 and $110 \mathrm{mg} / \mathrm{dL}$.

Blood samples for determination of plasma levels of cTnI, IL-6, IL-8, IL-10, TNF- $\alpha$, and elastase were taken after induction of anesthesia (T1), after revascularization but before protamine administration (T2), and 6 hours, 12 hours, and 24 hours after revascularization (T3, $\mathrm{T} 4$, and $\mathrm{T} 5$, respectively). The plasma fraction was isolated by centrifugation and stored at $-20^{\circ} \mathrm{C}$. Blood samples for determination of polymorphonuclear cell apoptosis (12 controls and 12 aprotinin-treated patients) were taken at the same time points except for T4 in EDTAcontaining tubes and were immediately sent to the laboratory for analysis.

The inflammatory cytokines IL-6, IL- 8 , and IL- 10 were measured by using cytometric bead array (CBA; BD Biosciences Europe, Erembodegem, Belgium) as described by the manufacturer. Acquisition of sample data was done by using a FACSort (BD Biosciences, San Jose, $\mathrm{CA}$ ), and results were generated using the BD CBA Analysis Software. The lowest detection limit for all cytokines was $1.25 \mathrm{pg} / \mathrm{mL}$.

Elastase was determined by sandwich enzyme-linked immunosorbent assay (Calbiochem; Merck Biosciences, Nottingham, UK). Samples were measured in duplicate. TNF- $\alpha$ was measured in an almost similar procedure to elastase by using the TNF- $\alpha$ EASIA kit purchased from Biosource Europe SA (Nivelles, Belgium). The lowest detection limits for elastase and TNF- $\alpha$ are $0.156 \mathrm{ng} / \mathrm{mL}$ and $14.7 \mathrm{pg} / \mathrm{mL}$, respectively. cTnI plasma levels were determined by a 1-step sandwich enzyme-linked immunosorbent assay technique and analyzed on a Dimension clinical chemistry analyzer (Dade Behring Inc, Brussels, Belgium).

Phosphatidylserine translocation from the inner to the outer cellular membrane is one of the first phenomenon to occur once apoptosis is induced. Annexin V is a naturally occurring high-affinity ligand for PS. Because translocation also occurs during necrosis, annexin $\mathrm{V}$ is not an absolute marker for apoptosis. Therefore, it is often used in conjunction with vital dyes, such as propidiumiodide, which bind to nucleic acids only when it can penetrate cells through a damaged membrane, thus distinguishing necrotic from apoptotic cells.

Briefly, blood was collected in a 3-mL EDTA tube and polymorphonuclear cells separated from the whole blood by using density gradient centrifugation. Obtained PMNs were resuspended in cold Hepes buffer at a concentration of $1 \times 10^{6} \mathrm{PMNs}$ per milliliter. Two hundred microliters of this cell suspension were used for analysis. Ten microliters of FITC-conjugated annexin V (stock: $20 \mu \mathrm{g} / \mathrm{mL}$ ) and 20 $\mu \mathrm{L}$ propidiumiodide (PI) (stock: $50 \mu \mathrm{L} / \mathrm{mL}$ ) (BD Biosciences Pharmingen, San Diego, CA) were added. After a gentle vortex, the PMNs were incubated for 15 minutes at $4{ }^{\circ} \mathrm{C}$ in the dark before flow cytometric analysis.
Apoptosis and cell death were measured by using a FACScan flow cytometer (Becton Dickinson, Erembodegem, Belgium) equipped with a 488 -nm argon-ion air-cooled laser $(15 \mathrm{~mW})$ and standard filter combination $(\mathrm{FL} 1=$ BP 530/30 nm, FL2 $=$ BP 585/42 nm, and FL3 $=$ LP $650 \mathrm{~nm}$ ) provided by the manufacturer. A minimum of 20,000 events were collected for each sample. The PMN population was gated on a forward-scatter versus side-scatter dot plot, and quadrant statistics were used on an annexin V versus PI dot plot to express apoptotic (annexin $\mathrm{V}^{\text {pos }} / \mathrm{PI}^{\text {neg }}$ ) and dead (annexin $\mathrm{V}^{\text {pos }} / \mathrm{PI}^{\mathrm{pos}}$ ) PMNs as fraction of the total number of PMNs. To calculate absolute numbers, these fractions were multiplied by the absolute PMN count obtained by a Micros 60 hematology analyzer (ABX Diagnostics, Deurne, Belgium). Persons involved in blood sample analysis (A.K., V.V.D., J.C., and M.D.) were not aware of the patient's allocation to either the control group or the aprotinin group.

Power analysis was performed on the primary outcome variable cTnI. A difference of $2 \mathrm{ng} / \mathrm{mL}$ was considered to be clinically important. For a power of 0.8 and $\alpha=0.05$, a sample size of 10 patients for each treatment group seemed to be sufficient.

Demographic data and data on blood loss and transfusion were analyzed by Student $t$ test. Because plasma levels of interleukins and elastase were not normally distributed, a Mann-Whitney $U$ test was used to look for differences between both groups. Apoptosis results were also not normally distributed and treated likewise. cTnI values, however, did show a normal distribution and were analyzed by using a repeated-measures analysis of variance approach. Statistical analysis was performed with Statistica, release 6.1 (StatSoft Inc, Tulsa, OK).

\section{RESULTS}

Demographic data for patients from both study groups are listed in Table 1. Distribution of age, weight, height, sex, and preoperative medication were not different between groups. Also, operating time, number of distal anastomoses (venous and arterial), incidence of intramural dissections, and heparin and protamine doses were not statistically different (Table 2). The number of patients who needed pharmacologic support during the study period was not statistically different between groups (Table 2; 9/25 control $v 7 / 25$ aprotinin group, $p=0.55$ ) nor was the total drug dose. Aprotinin administration did not

Table 1. Demographic and Preoperative Characteristics

\begin{tabular}{lcc}
\hline & Control & Aprotinin \\
\hline Age $(\mathrm{y})$ & $67.8( \pm 8.3)$ & $65.7( \pm 10.2)$ \\
Weight $(\mathrm{kg})$ & $80.6( \pm 9.3)$ & $76.8( \pm 13.9)$ \\
Height $(\mathrm{cm})$ & $171.5( \pm 9.4)$ & $168.6( \pm 8.7)$ \\
Sex ratio $(\mathrm{M} / \mathrm{F})$ & $20 / 5$ & $20 / 5$ \\
$\beta$-adrenergic blockers & $17 / 25$ & $16 / 25$ \\
Calcium channel blockers & $6 / 25$ & $7 / 25$ \\
ACE inhibitors & $8 / 25$ & $5 / 25$ \\
AT II & $3 / 25$ & $2 / 25$ \\
Nitrates & $16 / 25$ & $15 / 25$ \\
Diuretics & $3 / 25$ & $3 / 25$ \\
Bronchodilating agents & $0 / 25$ & $1 / 25$ \\
Acetylsalicylic acid & $19 / 25$ & $18 / 25$ \\
LMWH & $6 / 25$ & $7 / 25$ \\
Statins & $19 / 2$ & $15 / 25$ \\
\hline
\end{tabular}

NOTE. Data are expressed as mean \pm SD. Patient numbers are indicated for sex ratio and preoperative medication. No significant differences were found between groups.

Abbreviations: AT II, angiotensin II inhibitors; LMWH, low-molecular-weight heparins. 
Table 2. Operative and Postoperative Characteristics

\begin{tabular}{lccc}
\hline & Control & Aprotinin & $p$ Value \\
\hline Operating time (min) & $260.9( \pm 53.5)$ & $242.4( \pm 51.1)$ & 0.24 \\
Distal anastomoses & $2.9( \pm 1.17)$ & $2.8( \pm 1.30)$ & 0.57 \\
$\quad$ Arterial & $2.3( \pm 1.14)$ & $2.0( \pm 1.12)$ & 0.32 \\
$\quad$ Venous & $0.6( \pm 0.7)$ & $0.7( \pm 1.12)$ & 0.65 \\
Intramural dissection & $2 / 25$ & $3 / 25$ & 0.64 \\
Heparin dose (IU/kg) & $386.2( \pm 169.6)$ & $383.5( \pm 133.8)$ & 0.63 \\
Protamine dose (IU/kg) & $306.5( \pm 93.4)$ & $308.5( \pm 105.6)$ & 0.64 \\
Pharmacol. support & $9 / 25$ & $7 / 25$ & 0.55 \\
Total blood loss (mL) & $2132.2( \pm 1487.5)$ & $901.3( \pm 514.6)$ & 0.00029 \\
Washed reinfused blood & & & \\
$\quad$ per patient $(\mathrm{mL})$ & $242.0( \pm 292.0)$ & $41.6( \pm 123.3)$ & 0.0028 \\
Units packed cells per & & & \\
$\quad$ patient & $1.3( \pm 1.34)$ & $0.44( \pm 0.77)$ & 0.0065 \\
CRP (mg/dL) & & & \\
$\quad$ Preoperative & $4.34( \pm 5.04)$ & $3.93( \pm 5.10)$ & 0.8 \\
24-h postoperative & $67.54( \pm 27.88)$ & $64.62( \pm 31.87)$ & 0.76 \\
Serum creatinine (mg/dL) & & & \\
Preoperative & $1.22( \pm 0.20)$ & $1.17( \pm 0.24)$ & 0.45 \\
24-h postoperative & $1.23( \pm 0.67)$ & $1.09( \pm 0.27)$ & 0.35 \\
\hline
\end{tabular}

NOTE. Data are expressed as mean \pm SD. Patient numbers are indicated for intramural dissection and pharmacologic support.

Abbreviation: CRP, C-reactive protein.

influence plasma $\mathrm{C}$-reactive protein levels or serum creatinine levels throughout the study period (Table 2).

Plasma cTnI results for patients randomized to aprotinin or control group are shown in Figure 1. There were no differences in cTnI between groups at baseline (T1). Patients randomized to the aprotinin group did have a significantly lower cTnI concentration after revascularization $(\mathrm{T} 2, p=0.04)$ and at 6 hours $(\mathrm{T} 3, p=0.004)$ and 24 hours $(\mathrm{T} 5, p=0.03)$ after revascularization compared with controls.

The number of apoptotic PMN cells in both control and aprotinin groups are shown in Figure 2. The number of apoptotic PMN cells was lower in the aprotinin group at revascularization $(\mathrm{T} 2, p=0.04)$ and 6 hours after revascularization (T3, $p=0.04)$ compared with the controls. The absolute number of white blood cells and PMN cells did not differ between groups at any time point (data not shown).

Plasma levels of IL-6, $-8,-10$; TNF- $\alpha$; and elastase for both control and treatment groups are shown in Figure 3. For reasons of scaling nonoutlier ranges are represented by the whiskers. Full ranges are given in Table 3. In both groups, interleukin and elastase plasma levels increased from baseline for each time point throughout the study period. Peak levels of IL-6, IL-8, and TNF- $\alpha$ were observed at T3, whereas IL-10 peak levels occurred at T4. Plasma elastase levels gradually increased during the study period. No significant difference was found in the plasma concentrations of one of the inflammatory mediators tested between both groups. Blood loss during the study period was significantly lower in the aprotinin-treated patients compared with the controls (901 mL mean $v 2,131 \mathrm{~mL}$ mean, $p=$ $0.0003)$. The volume of reinfused, cell-saved blood was $41 \mathrm{~mL}$ per patient in the aprotinin group versus $242 \mathrm{~mL}$ per patient in the control group (mean per patient, $p=0.003$ ). The use of packed red cells was significantly reduced in aprotinin-treated subjects $(p=0.0065)$. One patient from the control group needed re-exploration for bleeding.

\section{DISCUSSION}

The authors report that perioperative aprotinin administration during $\mathrm{OPCAB}$ surgery resulted in reduced cTnI plasma levels up to 24 hours postoperatively, indicating reduced myocardial damage in the aprotinin-treated group. These results are in accordance with prior laboratory and clinical studies suggesting that aprotinin reduces the extent of myocardial damage caused by ischemia-reperfusion injury during CPB-supported surgery. ${ }^{20-24}$ More recently, reduced myocardial damage during OPCAB surgery was reported in patients receiving aprotinin. ${ }^{25,26}$ Considerable concern exists regarding graft patency and graft survival when antifibrinolytics are used during coronary bypass surgery. However, several meta-analyses studying on-pump CABG did not find any evidence supporting this concern. ${ }^{27,28}$ This is in agreement with the present observation in OPCAB surgery that aprotinin treatment resulted in reduced cTnI concentrations. In contrast, a recently published multicenter observational study showed an increased risk for renal failure, stroke, and cardiac failure associated with the use of aprotinin in CABG surgery. ${ }^{29}$ However, caution must be used when interpreting the results of the study by Mangano et al. ${ }^{29}$ A major limitation of the study is the absence of randomization resulting in an almost 2-fold higher incidence of complex surgery in the aprotinin group.

A possible mechanism was reported very recently by Khan and coworkers. ${ }^{30}$ They found that aprotinin treatment resulted in the preservation of coronary endothelial adherens junctions resulting in reduced tissue edema after regional ischemia and cardioplegic arrest. Another possible way of cardioprotection relies on aprotinin's ability to block the PAR-1 receptor. This

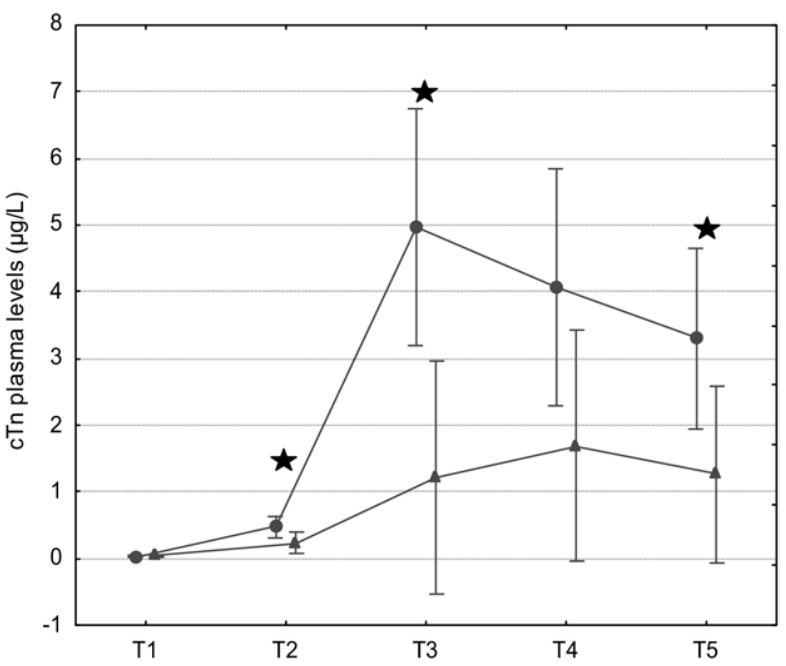

Fig 1. Time course of cTnl plasma levels in both control and aprotinin groups. Data are expressed as mean \pm standard deviation. T1-T5 indicate time points; see text for explanation. Circles: control group; triangles: aprotinin group; $\star$, significant difference between aprotinin group and control group ( $p$ values: T2 $=0.04$, T3 $=0.004$, and $\mathrm{T} 5=0.03$ ). 
Fig 2. Evolution of absolute number of apoptotic PMN cells in the control and aprotinin groups. Solid boxes indicate median; open boxes indicate 25th and 75th percentiles. Whiskers indicate range. T1, T2, T3, and $\mathrm{T} 5$ indicate time points; see text for explanation. At T2 and T3, numbers of apoptotic PMN cells are significantly different between groups $(p=0.04$ for both time points).
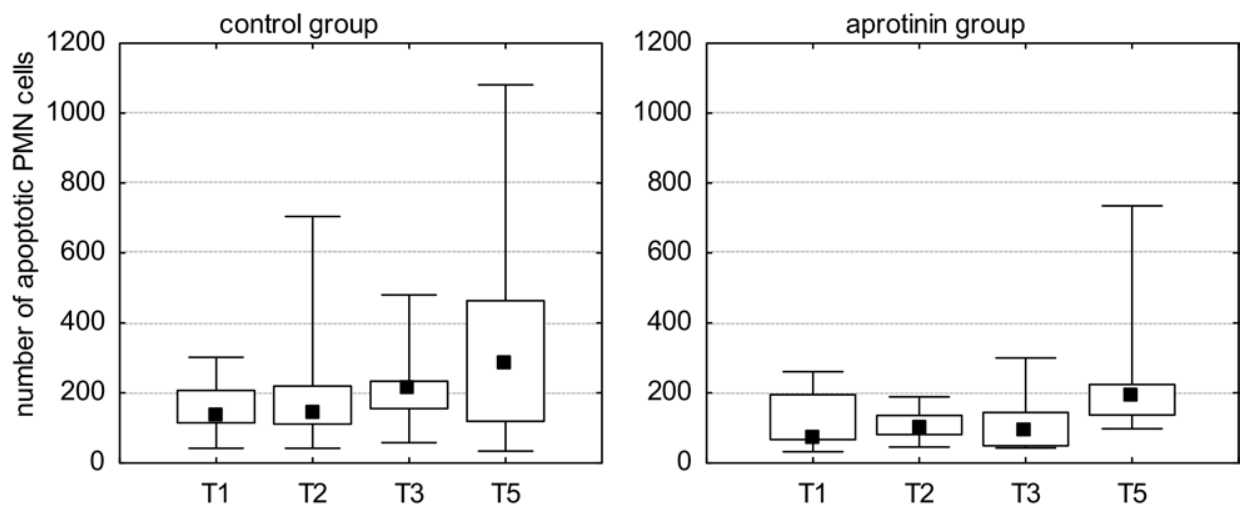

has recently been described to occur in patients undergoing cardiothoracic surgery. ${ }^{31}$ This PAR-1 receptor is the classic thrombin receptor, and its activation converts endothelial cells into a proinflammatory state with subsequent recruitment and activation of PMN cells. Recently, Khan et $\mathrm{al}^{32}$ showed that aprotinin inhibits protease-dependent platelet aggregation and thrombosis, which was, at least in part, mediated by PAR-1 inhibition. Platelet aggregation is associated with the release of proinflammatory mediators, and hence reduced platelet aggregation may contribute to a reduced local proinflammatory status. Poston et $\mathrm{al}^{25}$ recently suggested that aprotinin might have an antithrombotic action in addition to its antifibrinolytic effect. The potential anti-inflammatory actions of aprotinin could also contribute to a reduced myocardial invasion of activated granulocytes. The exact mechanism by which aprotinin reduces the perioperative cTnI release in OPCAB surgery is currently unknown and needs further investigation. Finally, this study adds to the fact that aprotinin use in primary cardiac surgery appears to be safe with regard to graft patency.

In this study, the authors describe, for the first time, an antiapoptotic effect of aprotinin on PMN cells in vivo. Trevani et $\mathrm{al}^{33}$ previously showed that aprotinin was able to prevent protease-induced apoptosis of neutrophils in vitro. They did not study whether this effect was mediated through inactivation of the proteases or by inhibition of PAR-1 activation. It has been shown that apoptosis of PMN cells is an important phenomenon in the termination of acute inflammatory processes. In addition, the occurrence of apoptotic resistance in PMN cells has been shown in patients suffering from SIRS and after major surgery. ${ }^{7,34}$ In view of the potential anti-inflammatory actions of aprotinin, it was expected that aprotinin would rather enhance PMN cell apoptosis. Nonetheless, the opposite was observed. Apparently aprotinin further enhanced apoptotic resistance in PMN cells after cardiac surgery. Whether this antiapoptotic effect of aprotinin further contributes to the development of systemic inflammation by prolonging the lifespan of PMN cells is currently unknown. These findings are interesting in view of the study by Mangano et $\mathrm{al}^{29}$ reporting an increased incidence of serious side effects when aprotinin is used during CABG surgery. It could be possible that the aprotinin-induced increased life span of PMN cells constitutes a contributing factor in the increased incidence of serious postoperative morbidity and hence aprotinin would have opposing effects.
As shown in the results section, no significant modulatory effect of aprotinin was observed on the plasma levels of the studied inflammatory mediators. Many, but not all, studies in CPB-supported cardiac surgery have provided evidence for an anti-inflammatory action of aprotinin. ${ }^{11-19,35-37}$ There seems to be conflicting evidence as to the presumed anti-inflammatory actions of aprotinin. On a theoretical basis and largely confirmed by in vitro investigations, aprotinin has potential antiinflammatory actions. ${ }^{1-6}$ However, in the clinical setting, the picture is less clear. No literature data with regard to the potential anti-inflammatory action of aprotinin during $\mathrm{OPCAB}$ surgery are available thus far. Because inflammation also occurs in OPCAB surgery, be it less pronounced compared with on-pump $\mathrm{CABG}$ surgery, $\mathrm{OPCAB}$ patients remain at risk for a complicated postoperative course. ${ }^{8-10}$ In this study in OPCAB patients, the authors failed to show an effect of aprotinin on the inflammatory mediators tested. It may be that the absence of contact activation in the OPCAB procedure makes the antiinflammatory action of aprotinin less pronounced. It is well known that one of the major pathways of aprotinin action is through inhibition of contact activation. A major limitation of the present study is that only a subset of inflammatory mediators has been determined at the expense of others such as those involved in the complement pathway. Also, the effect of cell saving on the plasma levels of inflammatory mediators is not well characterized. Two studies report more than $90 \%$ elimination of platelets, heparin, and proteins by the Autolog device. ${ }^{38,39}$ These studies report different elimination ratios for leukocytes of $78 \%$ and $91 \%$. The higher blood loss in the control group and consequently the more frequent washing of shed blood in this group may have diminished the total load of inflammatory mediators, masking the anti-inflammatory effect of aprotinin. This reduced mediator load upon washing of suctioned blood has also been suggested by other authors. ${ }^{40}$

In addition, the authors confirmed an important reduction in blood loss and use of allogeneic blood transfusion in patients receiving aprotinin. This blood-sparing effect has been shown in major surgery for at least 2 decades and has recently been confirmed during OPCAB surgery as well. ${ }^{25,41}$

From this study, it can be concluded that perioperative aprotinin administration in patients undergoing OPCAB reduces the perioperative $\mathrm{cTnI}$ release and hence may confer cardioprotection. The clinical significance of this observation is currently not known nor is the mechanism by which aprotinin evokes this 

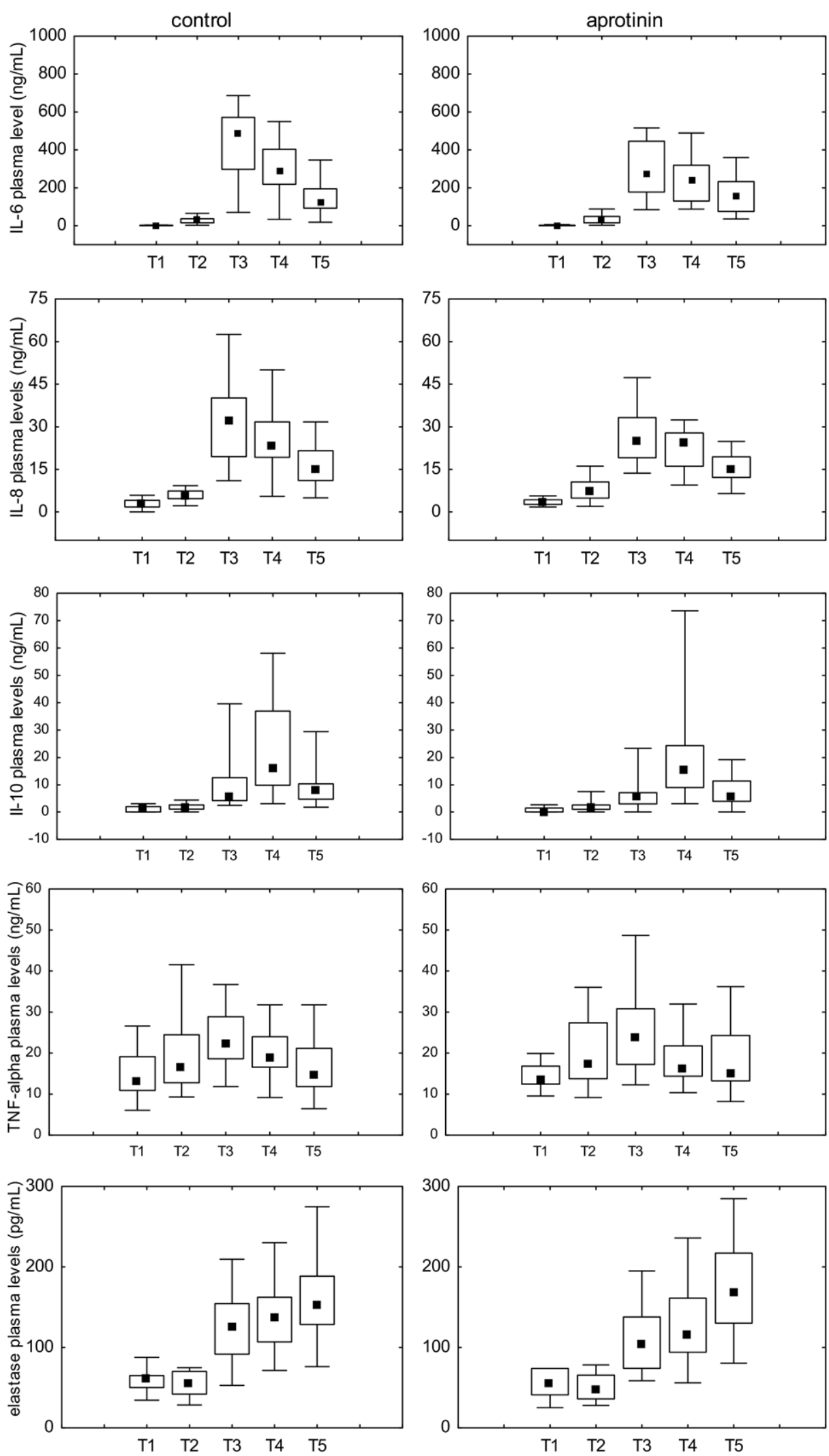

Fig 3. Evolution over time of IL-6, $-8,-10$, TNF- $\alpha$, and elastase plasma levels in both control and aprotinin groups. Solid boxes indicate median; open boxes indicate 25th and 75th percentiles. Whiskers indicate nonoutlier range. T1-T5 indicate time points; see text for explanation. 
Table 3. Ranges of IL-6, IL-8, IL-10, TNF- $\alpha$, and Elastase Plasma Levels in Both Control and Treatment Groups and $p$ Values Are Shown

\begin{tabular}{|c|c|c|c|c|c|}
\hline & $\mathrm{T} 1$ & $\mathrm{~T} 2$ & T3 & T4 & T5 \\
\hline \multicolumn{6}{|l|}{ IL-6 (ng/mL) } \\
\hline Control & $1.10-6.30$ & $3.0-65.50$ & $70.40-2,112$ & 33.8-991 & $19-691$ \\
\hline$p$ value & 0.873 & 0.424 & 0.107 & 0.196 & 0.776 \\
\hline \multicolumn{6}{|l|}{ IL-8 (ng/mL) } \\
\hline Control & $1.30-5.90$ & $2.20-9.30$ & $11.0-156.3$ & $5.50-91.50$ & $5.0-52.30$ \\
\hline$p$ value & 0.184 & 0.051 & 0.141 & 0.168 & 0.422 \\
\hline \multicolumn{6}{|l|}{ IL-10 (ng/mL) } \\
\hline Control & $1.40-3.10$ & $1.10-4.40$ & $2.50-39.60$ & $3.10-58.10$ & $1.80-29.40$ \\
\hline Aprotinin & $1.20-2.70$ & $1.00-7.50$ & $1.70-23.30$ & $3.10-73.60$ & $2.60-19.20$ \\
\hline$p$ value & 0.092 & 0.682 & 0.123 & 0.347 & 0.588 \\
\hline \multicolumn{6}{|c|}{ TNF- $\alpha(\mathrm{ng} / \mathrm{mL})$} \\
\hline Control & 6.09-44.39 & $9.30-41.57$ & $11.86-176.45$ & $9.20-89.97$ & 6.47-68.88 \\
\hline$p$ value & 0.676 & 0.987 & 0.699 & 0.440 & 0.573 \\
\hline \multicolumn{6}{|c|}{ Elastase $(\mathrm{pg} / \mathrm{mL})$} \\
\hline Control & $34.40-135.50$ & $28.30-261.80$ & $52.80-1095.60$ & $71.40-230.10$ & $76.10-397.00$ \\
\hline Aprotinin & $25.00-347.10$ & $28.00-167.00$ & $58.60-304.00$ & $55.90-747.20$ & $80.20-1269.00$ \\
\hline$p$ value & 0.120 & 0.334 & 0.286 & 0.546 & 0.261 \\
\hline
\end{tabular}

effect. In addition, an antiapoptotic effect of aprotinin was seen on PMN cells without any effect on the plasma levels of several inflammatory mediators. The authors also confirmed the blood- saving effect of aprotinin in patients undergoing OPCAB surgery. Further clinical trials are needed to explore whether aprotinin administration in OPCAB patients is clinically beneficial.

\section{REFERENCES}

1. Schini-Kerth VB, Boese M, Busse R, et al: N- $\alpha$-Tosyl-L-Lysine chloromethylketone prevents expression of iNOS in vascular smooth muscle by blocking activation of NF- $\kappa$ B. Arterioscler Thromb Vasc Biol 17:672-679, 1997

2. Poullis M, Manning R, Laffan M, et al: The antithrombotic effect of aprotinin: Actions mediated via the protease activated receptor 1 . J Thorac Cardiovasc Surg 120:370-378, 2000

3. Freise J, Magerstedt P, Schmid K: Inhibition of phospholipase A2 by gabexate mesilate, camostate and aprotinin. Enzyme 30:209-212, 1983

4. Asimakopoulos G, Taylor KM, Haskard DO, et al: Inihibition of neutrophil L-selectin shedding: A potential anti-inflammatory effect of aprotinin. Perfusion 15:495-499, 2000

5. Asimakopoulos G, Lidington EA, Mason J, et al: Effect of aprotinin on endothelial cell activation. J Thorac Cardiovasc Surg 122:123128,2001

6. Harmon D, Shorten WLG: The effect of aprotinin on hypoxiareoxygenation-induced changes in neutrophil and endothelial function. Eur J Anesthesiol 21:973-979, 2004

7. Jimenez MF, Watson RW, Parodo J, et al: Dysregulated expression of neutrophil apoptosis in the systemic inflammatory response syndrome. Arch Surg 132:1263-1269, 1997

8. Al-Ruzzeh S, Hoare G, Marczin N, et al: Off-pump coronary artery bypass surgery is associated with reduced neutrophil activation as measured by the expression of $\mathrm{CD} 11 \mathrm{~b}$ : A prospective randomized study. Heart Surg Forum 6:89-93, 2003

9. Okubo N, Hatori N, Ochi $\mathrm{M}$, et al: Comparison of mRNA expression for inflammatory mediators in leukocytes between on-pump and off-pump coronary artery bypass grafting. Ann Thorac Cardiovasc Surg 9:43-49, 2003

10. Schulze C, Conrad N, Schutz A, et al: Reduced expression of systemic proinflammatory cytokines after off-pump versus conven- tional coronary artery bypass grafting. Thorac Cardiovasc Surg 48:364-369, 2000

11. Hill GE, Pohorecki R, Alonso A, et al: Aprotinin reduces interleukin-8 production and lung neutrophil accumulation after coronary artery bypass. Anesth Analg 83:696-700, 1996

12. Diego RP, Mihalakakos PJ, Hexum TD, et al: Methylprednisolone and full-dose aprotinin reduce reperfusion injury after cardiopulmonary bypass. J Cardiothorac Vasc Anesth 11:29-31, 1997

13. Hill GE, Diego RP, Stammers AH, et al: Aprotinin enhances the endogenous release of interleukin-10 after cardiac operations. Ann Thorac Surg 65:66-69, 1998

14. Bruda NL, Hurlbert BJ, Hill GE: Aprotinin reduces nitric oxide production in vitro and in vivo in a dose-dependent manner. Clin Sci 94:505-509, 1998

15. Tassani P, Augustin N, Barankay A, et al: High-dose aprotinin modulates the balance between proinflammatory and anti-inflammatory responses during coronary artery bypass graft surgery. J Cardiothorac Vasc anesth 14:682-686, 2000

16. Isbir CS, Dogan R, Demircin M, et al: Aprotinin reduces the IL-8 after coronary artery bypass grafting. Cardiovasc Surg 9:403-406, 2001

17. Greilich PE, Okada K, Latham P, et al: Aprotinin but not $\epsilon$-aminocaproic acid decreases interleukin-10 after cardiac surgery with extracorporeal circulation. Circulation 104:I265-I269, 2001 (suppl I)

18. Harig F, Hohenstein B, von der Emde J, et al: Modulating IL-6 and IL-10 levels by pharmacologic strategies and the impact of different extracorporeal circulation parameters during cardiac surgery. Shock 16:33-38, 2001 (suppl I)

19. Greilich PE, Brouse CF, Whitten CW, et al: Antifibrinolytic therapy during cardiopulmonary bypass reduces proinflammatory cytokine levels: A randomized, double-blind, placebo-controlled study of epsilon-aminocaproic acid and aprotinin. J Thorac Cardiovasc Surg 126:1498-1503, 2003 
20. Gurevitch J, Barak J, Hochhauser E, et al: Aprotinin improves myocardial recovery after ischemia and reperfusion. J Thorac Surg 108:109-118, 1994

21. Pruefer D, Buerke U, Khalil M, et al: Cardioprotective effects of the serine protease inhibitor aprotinin after regional ischemia and reperfusion on the beating heart. J Thorac Cardiovasc Surg 124:942949, 2002

22. Hendrikx M, Rega F, Jamaer L, et al: $\mathrm{Na}+/ \mathrm{H}+$-exchange inhibition and aprotinin administration: Promising tools for myocardial protection during minimally invasive CABG. Eur J Cardiothorac Surg 19:633-639, 2001

23. Wendel HP, Heller W, Michel J, et al: Lower cardiac troponin T levels in patients undergoing cardiopulmonary bypass and receiving high-dose aprotinin therapy indicate reduction in perioperative myocardial damage. J Thorac Cardiovasc Surg 109:1164-1172, 1995

24. Hendrikx M, Jiang H, Gutermann H, et al: Release of cardiac troponin I in antegrade crystalloid versus cold blood cardioplegia. J Thorac Cardiovasc Surg 118:452-459, 1999

25. Poston RS, White C, Gu J, et al: Aprotinin shows both hemostatic and antithrombotic effects during off-pump coronary artery bypass grafting. Ann Thorac Surg 81:104-111, 2006

26. Vanek T, Jares M, Fajt R, et al: Fibrinolytic inhibitors in off-pump coronary surgery: A prospective, randomized, double-blind TAP study (tranexamic acid, aprotinin, placebo). Eur J Cardiothorac Surg 28:563-568, 2005

27. Levi M, Cromheecke ME, de Jonge E, et al: Pharmacological strategies to decrease excessive blood loss in cardiac surgery: A metaanalysis of clinically relevant endpoints. Lancet 354:1940-1947, 1999

28. Sedrakyan A, Treasure T, Elefteriades JA: Effect of aprotinin on clinical outcomes in coronary artery bypass graft surgery: A systematic review and meta-analysis of randomized clinical trials. J Thorac Cardiovasc Surg 128:442-448, 2004

29. Mangano DT, Tudor IC, Dietzel C: The risk associated with aprotinin in cardiac surgery. N Engl J Med 354:353-365, 2006
30. Khan TA, Bianchi C, Araujo E, et al: Aprotinin preserves cellular junctions and reduces myocardial edema after regional ischemia and cardioplegic arrest. Circulation 112:I196-I201, 2005 (suppl)

31. Day JR, Punjabi PP, Randi AM, et al: Clinical inhibition of the seven-transmembrane thrombin receptor (PAR1) by intravenous aprotinin during cardiothoracic surgery. Circulation 110:2597-2600, 2004

32. Khan TA, Bianchi C, Voisine P, et al: Aprotinin inhibits protease-dependent platelet aggregation and thrombosis. Ann Thorac Surg 79:1545-1550, 2005

33. Trevani AS, Andonegui G, Giordano M, et al: Neutrophil apoptosis induced by proteolytic enzymes. Lab Invest 74:711-721, 1996

34. Fanning NF, Porter J, Shorten GD, et al: Inhibition of neutrophil apoptosis after elective surgery. Surgery 126:527-534, 1999

35. Defraigne JO, Pincemail J, Larbuisson R, et al: Cytokine release and neutrophil activation are not prevented by heparin-coated circuits and aprotinin administration. Ann Thorac Surg 69:1084-1091, 2000

36. Schmartz D, Tabardel Y, Preiser JC, et al: Does aprotinin influence the inflammatory response to cardiopulmonary bypass in patients? J Thorac Cardiovasc Surg 125:184-190, 2003

37. Wei M, Kuukasjarvi P, Laurikka J, et al: Pump prime aprotinin fails to limit proinflammatory cytokine release after coronary artery bypass surgery. Scand Cardiovasc J 35:50-54, 2000

38. Serrick CJ, Scholz M, Melo A, et al: Quality of red blood cells using autotransfusion devices: A comparative analysis. J Extra Corpor Technol 35:28-34, 2003

39. Geiger P, Platow K, Bartl A, et al: New developments in autologous transfusion systems. Anaesthesia 53:32-35, 1998 (suppl 2)

40. Svenmarker S, Engstrom KG: The inflammatory response to recycled pericardial suction blood and the influence of cell-saving. Scand Cardiovasc J 37:158-164, 2003

41. Englberger L, Markart P, Eckstein FS, et al: Aprotinin reduces blood loss in off-pump coronary artery bypass (OPCAB) surgery. Eur J Cardiothorac Surg 22:545-551, 2002 